1 Van Vliet PD, Burchell HB, Titus JL. Focal myocarditis associated with pheochromocytoma. N Engl f Med 1966;274:1102-8.

${ }^{2}$ Pelkonen R, Pitkanen E. Unusual electrocardiographic changes in pheochromocytoma. Acta Med Scand 1963;173:41-4.

${ }^{3}$ Radtke WE, Kazmier FJ, Rutherford BD, Sheps SG. Cardiovascular complications of pheochromocytoma crisis. Am f Cardiol 1975;35:701-5.

${ }^{4}$ Hausmann VM, Getzowa S. Adenoma of organ of Zuckerkandle. Schweiz Med Woch 1922;52:889-92.

(Accepted 1 March 1983)

Regional Medical Cardiology Centre, Royal Victoria Hospital, Belfast

P MCGONIGLE, MB, MRCP, registrar

$S$ W WEBB, MD, MRCP, consultant cardiologist

A A J ADGEY, MD, FRCP, consultant cardiologist

Correspondence to: Dr A A J Adgey.

\section{Measles serology in children with a history of measles in early life}

Since the introduction of routine measles vaccination in 1968 the yearly uptake of the vaccine in England and Wales has not exceeded $54 \% .^{1}$ The failure to protect against this disease is reflected in the 139485 cases reported to the Office of Population Censuses and Surveys in $1980 .^{2}$

In many children who were admitted to a north London district hospital and in whom there was a history of measles before the age of 12 months we found that the duration of the rash was often stated to be only two or three days and a history of cough frequently absent or very mild. Hence we were led to question the diagnosis of measles in these children. Since a history of measles is one of the reasons for withholding measles vaccine, we decided to measure the serum measles antibody titres in children with such a history.

\section{Patients, methods, and results}

With informed consent in each case, 80 children aged 6-36 months who were seen at St Ann's General Hospital, London, after April 1980 were examined serologically for measles haemagglutination inhibition antibodies. The age at which measles was stated to have occurred was recorded. Clinic records confirmed that none of the children had received measles vaccine. According to the mothers, 76 of the children had had their measles diagnosed by either the family doctor or the clinic doctor; in four cases the parents or grandparents had made the diagnosis.

Measles was stated to have occurred before the first birthday in 53 children, and in nine of these before the age of 6 months. The table gives the results. Of children whose measles was stated to have occurred before 12 months of age, over two thirds were seronegative (haemagglutination inhibition antibody titre $<1 / 8$ ). Also of children whose measles was reported after the age of 12 months, just under one third were seronegative.

Number and percentage of seronegative children according to age at which measles was reported

\begin{tabular}{ccc}
\hline Age measles reported (months) & No examined & No (\%) seronegative* \\
\hline $4-12$ & 53 & $36(67.9)$ \\
$13-24$ & 27 & $8(29.6)$
\end{tabular}

${ }^{*}$ Measles haemagglutination inhibition antibody titre $1 / 8$

\section{Comment}

Measles is a not inconsequential infection, with a moderate to high fever, conjunctivitis, a blotchy rash lasting three or more days, and a troublesome cough. The presence of Koplik's spots is pathognomonic. The clinical diagnosis should not therefore be difficult in most cases, and measles should not be confused with other infections accompanied by a morbilliform rash in early childhood. These are chiefly caused by enteroviruses; in these infections Koplik's spots are not seen, the rash often lasts for less than three or four days, and coughing is seldom protracted or severe. It was surprising that the diagnosis of measles in children under 1 year and even in almost one third of children aged 12-24 months was so often incorrect as judged by the serological evidence.

As was found by one of us in an earlier study, ${ }^{3}$ these findings are relevant in attempts to prevent measles by vaccination in Britain. In the earlier study one of the most frequent reasons for failure to be given vaccine was the history of measles before the 15-18 months of age at which the vaccine was due to be given. There is no evidence that measles vaccine is harmful to a child who has had the disease (S Krugman, personal communication, 1981). We therefore suggest that measles vaccine should be administered to children irrespective of a history of the disease in the first year of life. If there is hesitation to give vaccine because of an alleged history of measles the uncertainty of such a clinical diagnosis can be pointed out to the parents and antibody testing offered to clarify things.

We thank Dr Christine Miller for helpful comments.

1 Department of Health and Social Security. Health and personal services statistics. London: HMSO, 1980.

${ }^{2}$ Office of Population Censuses and Surveys. Infectious diseases, 1980. London: OPCS, 1980.

${ }^{3}$ Adjaye N. Measles immunization: some factors affecting non-acceptance of vaccine. Public Health (London) 1981 ;95:185-8.

(Accepted 22 February 1983)

St Mary's Medical School, London W2

NELLIE ADJAYE, MB, MRCP, lecturer in community paediatrics

Haringey Health District, London

A AZAD, MB, BS, clinical medical officer

MARGARET FOSTER, $\mathrm{MB}, \mathrm{BCH}$, clinical medical officer

The Hospital for Sick Children, Great Ormond Street, London WC1N 3JH

W C MARSHALL, MD, FRACP, consultant paediatrician

HELEN DUNN, BSC, FIMLS, senior medical laboratory scientific officer

Correspondence and requests for reprints to: Dr W C Marshall.

\section{Therapeutic abortion and chlamydial infection}

Chlamydia trachomatis is a common cause of genital infection in both sexes. ${ }^{1}$ In women, symptomless colonisation of the cervix may be followed by an ascending infection and the development of pelvic inflammatory disease. We set out to determine the incidence of sexually transmitted infections (including Chl trachomatis infection) in a group of women attending this hospital for termination of pregnancy.

\section{Patients, methods, and results}

A group of women attending the day care unit of the department of gynaecology at this hospital requesting therapeutic abortion within the first seven weeks of pregnancy was studied. Patients who had received antimicrobial drugs during the previous three months were excluded, and a total of 89 entered the study.

Specimens were collected from the cervix before operation and cultured by standard procedures for Neisseria gonorrhoeae, Chl trachomatis, Mycoplasma hominis, and Ureaplasma urealyticum; and from the vagina for Trichomonas vaginalis and Candida spp. A specimen of blood was taken for serological

Micro-organisms isolated from lower genital tract of 89 women before termination of pregnancy

\begin{tabular}{lc}
\hline \multicolumn{1}{c}{ Micro-organisms } & No of patients infected \\
\hline Chl trachomatis alone & 1 \\
Chl trachomatis, $U$ urealyticum & 3 \\
Chl trachomatis, $U$ urealyticum, M hominis & 2 \\
Chl trachomatis, $U$ urealyticum, $M$ hominis, $N$ gonorrhoeae & 1 \\
$M$ hominis alone & 3 \\
$M$ hominis, $U$ urealyticum & 13 \\
$U$ urealyticum & 33 \\
vaginalis & 0 \\
Candida spp & 16 \\
\hline
\end{tabular}


tests for Chlamydia; these tests were performed by the whole inclusion immunofluorescence technique.

The table gives details of the micro-organisms isolated from the women. Serological tests showed IgG antibodies to Chlamydia at a titre of $\geqslant 1 / 64$ in all of seven women who yielded Chl trachomatis, and 60 of 82 women whose cultures grew no pathogens; the difference is not significant. IgM antibodies to Chlamydia at a titre of $\geqslant 1 / 8$ was present in four of the seven women with chlamydial infection and in 43 of the 82 with no chlamydial infection; again the difference is not significant.

\section{Comment}

Pelvic inflammatory disease occurs after first trimester therapeutic abortion in from $0.1 \%$ to $5.2 \%$ of unselected cases. ${ }^{2}$ Gonococcal infection was uncommon in our study, but cervical infection with Chl trachomatis, an important aetiological agent in pelvic inflammatory disease, was found in $8 \%$, and infection by $M$ hominis, which may also cause pelvic inflammatory disease, ${ }^{3}$ in $21 \%$ of 89 women.

Prospective studies have shown that nearly one quarter of women with chlamydial infection at the time of termination of pregnancy will subsequently develop pelvic inflammatory disease, and the management of this problem may be approached in two ways. An appropriate antibacterial agent could be given prophylactically to all women having abortions. A single dose of doxycycline $500 \mathrm{mg}$ has been advocated, ${ }^{4}$ but such a dose would be unlikely to eradicate $C h l$ trachomatis completely and treatment with a tetracycline for at least one week is necessary. This procedure would leave male sexual contacts unidentified, and therefore untreated, with the possibility of reinfection.

Alternatively, women attending for termination should be screened for $N$ gonorrhoeae, $C h l$ trachomatis, and possibly $M$ hominis before operation, and treated if found to be infected. At present, cell culture for Chl trachomatis is not widely available; serological tests on a single serum sample are, as shown in this study, unreliable for the diagnosis of Chl trachomatis, and paired serum samples are hardly practicable in the context of termination of pregnancy. The need for wider diagnostic facilities for Chl trachomatis has been advocated. ${ }^{5}$ Our findings have shown that the identification of this and other sexually transmitted microbes in women before termination of pregnancy would be a useful piece of preventive medicine. Further studies on antenatal populations, and on women attending for inpatient abortion, are in progress.

' Schachter J. Chlamydial infections. $N$ Engl $\mathcal{F}$ Med 1978;298:428-35, 490-5, 540-9.

2 Burkman RT. Culture and treatment results in endometritis following elective abortion. Am $\mathcal{F}$ Obstet Gynecol 1977;128:556-9.

${ }^{3}$ Mårdh P-A, Weström L. Tubal and cervical cultures in acute salpingitis with special reference to Mycoplasma hominis and T-strain mycoplasmas. Br $\mathcal{F}$ Vener Dis 1970;46:179-86.

${ }^{4}$ Brewer C. Prevention of infection after abortion with a supervised single dose of oral doxycycline. Br Med f 1980;281:780-1.

Richmond SJ, Oriel JD. Recognition and management of genital chlamydial infection. Br Med f 1978;ii:480-3.

(Accepted 28 February 1983)

\section{University College Hospital, London WC1E 6AU}

G L RIDGWAY, MD, MRCPATH, consultant microbiologist

G MUMTAZ, BSC, FIMLS, senior medical laboratory scientific officer

$\mathrm{R}$ A STEPHENS, MB, BS, clinical assistant, department of obstetrics and gynaecology

J D ORIEL, MD, consultant physician, department of genitourinary medicine

Correspondence to: Dr G L Ridgway, department of medical microbiology.

\section{Ultrasonic assessment of stress fractures}

Stress fractures are common in athletes and young military personnel. The wide range of possible sites and the varying frequency depending on the type of activity have been well described. ${ }^{12}$ Although there is increasing awareness of such fractures and their relation to overuse, early lesions may not be visible in conventional radiographs. Bone scintigraphy is a sensitive means of detection but may not be readily available. ${ }^{3}$ A chance finding, confirmed by others, ${ }^{4}$ that such stress lesions may be painful after therapeutic ultrasound prompted the following prospective study.

\section{Patients, methods, and results}

Thirty five consecutive patients (29 men; mean age 25 years, range 16-51) attending a sports injury clinic with a history and clinical findings suggestive of a stress fracture of the leg were included in the study. Three women with rheumatoid arthritis (ages 25,68 , and 70 ) and possible stress fractures related to osteoporosis and treatment with corticosteroids were also included. The operator using the ultrasound machine was unaware of the clinical and radiological findings. In addition we studied 17 patients ( 10 males; mean age 24 years, range 5-58) attending an accident department with radiologically confirmed recent fractures of a bone lying close to the skin surface. The operator was unaware that five young patients had greenstick fractures.

A control group of $17 \mathrm{fit}$, physically active subjects (11 men; mean age 26 years, range 16-45) agreed to describe sensations produced by the application of the standardised ultrasound over four typical sites of stress fracture in their right leg. These were over the lateral lower third of the fibula, the anteromedial aspect of the midshaft of the tibia, the anterior part of the medial tibial plateau, and the lateral aspect of the lower third of the femur.

After a trial of various frequencies continuous ultrasound at $0.75 \mathrm{MHz}$ was applied from a Sonacel Multiphon Mark II machine using a $3 \mathrm{~cm}$ head and a water based coupling medium. In those with possible or definite fractures the comparable site on the opposite side was also used to allow different sensations to be described. The intensity was gradually increased to a maximum of $2.0 \mathrm{~W} / \mathrm{cm}^{2}$ and a positive response defined as a very unpleasant sensation of intense pressure or pain, this usually occurring between 1.0 and $2 \cdot 0 \mathrm{~W} / \mathrm{cm}^{2}$

Only one of the 17 normal controls had a single, unexplained positive ultrasound reaction at the midtibial test site. None of the patients had positive reactions at the comparable, non-fracture site. Thus there was only one false positive response in 123 tests $(0.8 \%)$.

Ten of the 11 athletes with clear, plain radiographs but subsequently definite scintigraphic evidence of stress lesions had a positive ultrasound reaction, while all eight athletes with clear radiographs and negative findings on bone scintigraphy had negative ultrasound reactions (table). A further

Ultrasonic, radiological, and scintigraphic findings

\begin{tabular}{|c|c|c|c|c|c|c|}
\hline \multirow{3}{*}{$\begin{array}{l}\text { Ultrasound } \\
\text { reaction }\end{array}$} & \multicolumn{3}{|c|}{ Radiograph clear } & \multicolumn{3}{|c|}{ Radiological evidence of fracture } \\
\hline & \multicolumn{2}{|c|}{ Scintigraphy } & \multirow{2}{*}{$\begin{array}{l}\text { Follow } \\
\text { up } \\
\text { negative }\end{array}$} & \multirow{2}{*}{ Stress } & \multirow{2}{*}{ True } & \multirow{2}{*}{ Greenstick } \\
\hline & Positive & Negative & & & & \\
\hline $\begin{array}{l}\text { Positive } \\
\text { Negative }\end{array}$ & $\begin{array}{r}10 \\
1\end{array}$ & 8 & 10 & 7 & 11 & 5 \\
\hline
\end{tabular}

group of nine athletes and a younger patient with rheumatoid arthritis who had clear, plain radiographs and negative ultrasound reactions did not have bone scintigraphy; they were carefully followed up, however, often receiving therapeutic ultrasound and only slight restriction of activity for a presumed soft tissue lesion, and in no case did signs or symptoms of a definite stress fracture develop. These findings represent an accuracy of $96 \%$.

Seven athletes had radiologically confirmed stress fractures with negative ultrasound reactions. One sportsman and one patient with arthritis had true fractures with positive ultrasound reactions. Nine of the 12 patients with full fractures seen in the accident department had positive reactions while all five of those with greenstick fractures had negative reactions.

\section{Comment}

These results suggest that standardised application of $0.75 \mathrm{MHz}$ ultrasound may be helpful in the early diagnosis of stress fractures in sportsmen.

A stress fracture in a sportsman demands prompt reduction or stopping of the activity responsible, often for several weeks, while such advice is inappropriate and unwelcome to those without such lesions. Mimicking soft tissue lesions may respond to more active forms of treatment. Healing stress fractures shown clearly in initial radiographs and greenstick fractures are not painful with ultrasound, while most recent true fractures are. This difference has not been noted before, although others have observed that the rate of positive responses in true fractures decreases with time. ${ }^{5}$ These differences in response suggest that damaged periosteum may absorb continuous ultrasound energy with its conversion to heat and the development of pain, but that intact periosteum or periosteum involved in significant callus formation does not absorb this energy.

${ }^{1}$ Devas M. Stress fractures. Edinburgh: Churchill Livingstone, 1975. 